\title{
Non-convex block-sparse compressed sensing with coherent tight frames
}

\author{
Xiaohu Luo ${ }^{1}$ (D), Wanzhen Yang ${ }^{2}$, Jincai $\mathrm{Ha}^{3}$, Xing $\mathrm{Ai}^{4}$ and Xishan Tian ${ }^{*}$
}

\begin{abstract}
In this paper, we present a non-convex $\ell_{2} / \ell_{q}(0<q<1)$-analysis method to recover a general signal that can be expressed as a block-sparse coefficient vector in a coherent tight frame, and a sufficient condition is simultaneously established to guarantee the validity of the proposed method. In addition, we also derive an efficient iterative re-weighted least square (IRLS) algorithm to solve the induced non-convex optimization problem. The proposed IRLS algorithm is tested and compared with the $\ell_{2} / \ell_{1}$-analysis and the $\ell_{q}(0<q \leq 1)$-analysis methods in some experiments. All the comparisons demonstrate the superior performance of the $\ell_{2} / \ell_{q}$-analysis method with $0<q<1$.
\end{abstract}

Keywords: Block-sparse compressed sensing, Restricted isometry property, Restricted orthogonality property, Tight frames, $\ell_{2} / \ell_{q}$-analysis method

\section{Introduction}

Data compression and data recovery (maybe from its compressed observed data) are two crucial problems in many real-world applications, including information processing [1], machine learning [2], statistical inference [3], swarm intelligence $[4,5]$, and compressed sensing $(\mathrm{CS})[6,7]$. Among these applications, CS is particularly attractive since it provides insights into signal processing with significantly smaller samplings than classical signal processing approaches based on the Nyquist-Shannon sampling theorem.

CS was pioneered by Donoho [6] and Candès et al. [7] around 2006, and it has already captured lots of attention from researchers in a growing number of fields, including signal processing, machine learning, mathematical statistics, etc. A crucial concern in CS is to recover an unknown signal $\boldsymbol{f} \in \mathbb{R}^{\widetilde{n}}$ from its small set of linear measurements

$$
y=\Phi f,
$$

where $\boldsymbol{y} \in \mathbb{R}^{\widetilde{m}}$ is an observed signal vector and $\Phi \in \mathbb{R}^{\tilde{m} \times \tilde{n}}$ is a given measurement matrix with $\widetilde{m} \ll \widetilde{n}$.

Conventional CS heavily relies on techniques that can express signals as a few linear combination of base vectors

*Correspondence: nxdxtxs@163.com

${ }^{5}$ Institute of Ethnic Preparatory Education, NingXia University, Yinchuan

750002, China

Full list of author information is available at the end of the article from an orthogonal basis. However, in a large number of practical applications, the signals are not sparse in terms of an orthogonal basis, but in terms of an overcomplete and tight frame $[8,9]$. In such a scenario, one natural way to express $\boldsymbol{f}$ is to write $\boldsymbol{f}=\Psi \boldsymbol{x}$, where $\Psi \in \mathbb{R}^{\tilde{n} \times n}$ is a matrix with $\widetilde{n} \leq n$ whose $n$ columns form a tight frame, and $\boldsymbol{x} \in \mathbb{R}^{n}$ is sparse (or nearly sparse). In order to recover $\boldsymbol{f}$, a popular approach is using an $\ell_{1}$-synthesis method [10, $11]$, which first solves following problem:

$$
\min _{\boldsymbol{x} \in \mathbb{R}^{n}}\|\boldsymbol{x}\|_{1} \text { subject to } \boldsymbol{y}=\Phi \Psi \boldsymbol{x}
$$

to get the transform-based sparse coefficient vector $x^{\sharp}$, and then reconstruct the original signal $\boldsymbol{f}^{\sharp}$ by applying a synthesis operator $\Psi$ on $\boldsymbol{x}^{\sharp}$, i.e., $\boldsymbol{f}^{\sharp}=\Psi \boldsymbol{x}^{\sharp}$. Since the entries in $\Phi \Psi$ are correlated when $\Psi$ is highly coherent, $\Phi \Psi$ may no longer satisfy the required assumptions such as the restricted isometry property (RIP) and the mutual incoherence property (MIP) which have been widely used in conventional CS. Therefore, it is not easy to study the theoretical performance of the $\ell_{1}$-synthesis method.

Fortunately, there exists an alternative to the $\ell_{1}$ synthesis method called the $\ell_{1}$-analysis method $[10,12]$, which directly finds an estimator $\boldsymbol{f}^{\sharp}$ by solving following $\ell_{1}$-analysis problem:

$$
\min _{\boldsymbol{f} \in \mathbb{R}^{N}}\left\|\Psi^{T} \boldsymbol{f}\right\|_{1} \text { subject to } \boldsymbol{y}=\Phi \boldsymbol{f} .
$$


The $\ell_{1}$-analysis method has its roots in the analysis-style sparse representation $\boldsymbol{x}=\Psi^{T} \boldsymbol{f}$, and is different from the above-mentioned synthesis method, which is based on the synthesis-style sparse representation, i.e., $f=\Psi \boldsymbol{x}$. The existing literature has shown that there is a remarkable difference between the two methods despite their apparent similarity. For example, these two methods have totally different recovery conditions to guarantee robust recovery of any signal, and their ways to utilize the sparsity prior are also totally different. Please see $[10,13]$ and references therein for more details. To investigate the theoretical performance of the $\ell_{1}$-analysis method, Candès et al. [12] introduced the definition of $\Psi$-RIP: a measurement matrix $\Phi$ is said to satisfy the RIP adapted to $\Psi$ ( $\Psi$-RIP) with constant $\delta_{k}$ if

$$
\left(1-\delta_{k}\right)\|\Psi \boldsymbol{x}\|_{2}^{2} \leq\|\Phi \Psi \boldsymbol{x}\|_{2}^{2} \leq\left(1+\delta_{k}\right)\|\Psi \boldsymbol{x}\|_{2}^{2}
$$

holds for every vector $\boldsymbol{x} \in \mathbb{R}^{n}$ that is $k$-sparse, and establishes a sufficient condition related to $\Psi$-RIP for recovering general signals. In addition, they also demonstrated the efficiency of the $\ell_{1}$-analysis strategy with a large number of experiments based on real signals.

Different from the general case in CS that the transformbased coefficient vector $\boldsymbol{x}$ is sparse, some signals in the real world may exhibit additional sparse structures in terms of a fixed transform basis $\Psi$. Take for example the block-sparse structure, i.e., the non-zero elements of $x$ are assembled in a few fixed blocks, which is also our main concern in this paper. Such structured signals naturally arise in various applications. Prominent examples include DNA microarrays [14], color imaging [15], and motion segmentation [16]. Without loss of generality, we assume that there are $m$ blocks of size $d=n / m$ in $x$. Then, one can write any block-sparse vector $x \in \mathbb{R}^{n}$ as

$\boldsymbol{x}=[\underbrace{x_{1}, \cdots, \boldsymbol{x}_{d}}_{x[1]}, \underbrace{x_{d+1}, \cdots, \boldsymbol{x}_{2 d}}_{x[2]}, \cdots, \underbrace{\boldsymbol{x}_{n-d+1}, \cdots, \boldsymbol{x}_{n}}_{x[m]}]^{T}$,

where $\boldsymbol{x}[i]$ denotes the $i$ th block of $\boldsymbol{x}$. If $\boldsymbol{x}$ has at most $k$ non-zero blocks, i.e., $\|\boldsymbol{x}\|_{2,0} \leq k$, we refer to such a vector $\boldsymbol{x}$ as a block $k$-sparse signal. Accordingly, we can also write $\Psi \in \mathbb{R}^{\tilde{n} \times n}$ as

$\Psi=[\underbrace{\Psi_{1}, \cdots, \Psi_{d}}_{\Psi[1]}, \underbrace{\Psi_{d+1}, \cdots, \Psi_{2 d}}_{\Psi[2]}, \cdots, \underbrace{\Psi_{n-d+1}, \cdots, \Psi_{n}}_{\Psi[m]}]$,

where $\Psi_{i}$ with $i=1,2, \cdots, n$ and $\Psi[j]$ with $j=$ $1,2, \cdots, m$ are denoted by the $i$ th column vector and the $j$ th sub-block matrix of $\Psi$, respectively. Most current papers focus more on the conventional sparse or nearly sparse case in terms of $\Psi$. As one of some exceptions, Wang et al. [17] proposed an $\ell_{2} / \ell_{1}$-analysis method to investigate the recovery of block-sparse signals in terms of $\Psi$. Basing their theoretical analysis on block $\Psi$-RIP, which is a block version of $\Psi$-RIP that we will define in the next section, Wang et al. [17] also developed several sufficient conditions to guarantee robust recovery of general signals. For completeness, we present the $\ell_{2} / \ell_{1}$-analysis problem as follows

$$
\min _{\boldsymbol{f} \in \mathbb{R}^{N}}\left\|\Psi^{T} \boldsymbol{f}\right\|_{2,1}:=\sum_{i=1}^{m}\left\|\Psi[i]^{T} \boldsymbol{f}\right\|_{2} \text { subject to } \boldsymbol{y}=\Phi \boldsymbol{f},
$$

Obviously, when $d=1$, the $\ell_{2} / \ell_{1}$-analysis method will degenerate to the $\ell_{1}$-analysis method mentioned above.

Recently, the work of Chartrand et al. [18-20] has shown that the non-convex $\ell_{q}(0<q<1)$ method allows the exact recovery of sparse signals from a smaller set of linear measurement than that of the $\ell_{1}$ method, providing a new paradigm to study CS problems. In this paper, along with previous works on the non-convex $\ell_{q}(0<q<1)$ strategy, we first propose an $\ell_{2} / \ell_{q}$-analysis method with $0<q \leq 1$ to recover general signals that can be expressed as blocksparse signals in terms of $\Psi$. Our method is different from conventional CS methods, which only concern cases where the signals per se are sparse or block-sparse [18, 21-23], and also different from previous analysis methods [24-26], which only focus on the recovery of general signals that are expressed as non-block structured signals in terms of $\Psi$. Specifically, the proposed method can be described as:

$\min _{\boldsymbol{f} \in \mathbb{R}^{N}}\left\|\Psi^{T} \boldsymbol{f}\right\|_{2, q}:=\left\{\sum_{i=1}^{m}\left\|\Psi[i]^{T} \boldsymbol{f}\right\|_{2}^{q}\right\}^{1 / q}$ subject to $\boldsymbol{y}=\Phi \boldsymbol{f}$,

In many application problems, the observed signal $y$ may be polluted by a bounded noise $\boldsymbol{e}$, i.e., $\boldsymbol{y}=\Phi \boldsymbol{f}+\boldsymbol{e}$. So for the general situation, we have the model:

$$
\min _{\boldsymbol{f} \in \mathbb{R}^{N}}\left\|\Psi^{T} \boldsymbol{f}\right\|_{2, q} \text { subject to }\|\boldsymbol{y}-\Phi \boldsymbol{f}\|_{2} \leq \epsilon,
$$

where $\epsilon$ is the noise level. Secondly, for (1), we also establish a sufficient conditions for robust recovery of general signals. The obtained results associate two constants $\Psi$ RIC and $\Psi$-ROC in a block version with different $q \in$ $(0,1]$, and provide a series of selectable conditions for robust recovery via the $\ell_{2} / \ell_{q}$-analysis method. Finally, inspired by the ideas of $[21,27]$, we derive an iterative re-weighted least square (IRLS) algorithm to solve our $\ell_{2} / \ell_{q}$-analysis problem. Also, some experiments are conducted later that further demonstrate the efficiency of our $\ell_{2} / \ell_{q}$-analysis method with $0<q \leq 1$.

The rest of the paper is organized as follows. In Section 2, we first state three key definitions and then present our main theoretical results. In Section 3, we propose an IRLS algorithm to solve the $\ell_{2} / \ell_{q}$-analysis problem, and conduct some experiments to support the validity of our $\ell_{2} / \ell_{q}$-analysis method. Finally, the conclusion is addressed in Section 4. 


\section{Robust recovery for $\ell_{2} / \ell_{q}$-analysis problem}

In this section, we mainly establish a sufficient condition to robustly recover general signals that can be expressed as block-sparse vectors in terms of $\Psi$. Before presenting our main results, we first introduce several definitions that will be used later. We start with the introduction of two important definitions, which can also be found in many references such as [17].

Definition 1 Let $\Psi \in \mathbb{R}^{\tilde{n} \times n}$ with $\tilde{n} \leq n$ be a matrix whose $n$ columns form a tight frame. A measurement matrix $\Phi \in \mathbb{R}^{\widetilde{m} \times \widetilde{n}}$ is said to satisfy the block $\Psi-R I P$ condition with constant $\delta_{k \mid d}($ block $\Psi-R I C)$ if

$$
\left(1-\delta_{k \mid d}\right)\|\Psi \boldsymbol{x}\|_{2}^{2} \leq\|\Phi \Psi \boldsymbol{x}\|_{2}^{2} \leq\left(1+\delta_{k \mid d}\right)\|\Psi \boldsymbol{x}\|_{2}^{2}
$$

holds for every vector $\boldsymbol{x} \in \mathbb{R}^{n}$ that is block $k$-sparse.

Definition 2 The block $\Psi$-restricted orthogonality constant(block $\Psi$-ROC), denoted by $\theta_{\left(k_{1}, k_{2}\right) \mid d}$, is the smallest positive number that satisfies

$$
\left|\left\langle\Phi \Psi \boldsymbol{x}_{1}, \Phi \Psi \boldsymbol{x}_{2}\right\rangle-\left\langle\Psi \boldsymbol{x}_{1}, \Psi \boldsymbol{x}_{2}\right\rangle\right| \leq \theta_{\left(k_{1}, k_{2}\right) \mid d}\left\|\boldsymbol{x}_{1}\right\|_{2}\left\|\boldsymbol{x}_{2}\right\|_{2}
$$

for every $\boldsymbol{x}_{1}$ and $\boldsymbol{x}_{2}$ such that $\boldsymbol{x}_{1}$ and $\boldsymbol{x}_{2}$ are block $k_{1}$-sparse and block $k_{2}$-sparse, respectively.

It is easy to see that if one sets $\Psi$ to be the identity matrix of size $\tilde{n} \times \tilde{n}$, then the above-mentioned definitions will be reduced to the well-known block-RIC and blockROC definitions. Furthermore, if one sets the block size $d=1$, then we will get the classical RIC and ROC definitions. Obviously, block-RIC and block-ROC definitions, together with RIC and ROC definitions, are just two special cases of Definitions 1 and 2. In addition, we also need the following definition, which also plays a key role in our theorem.

Definition 3 Given $\boldsymbol{x} \in \mathbb{R}^{n}$, we denote the best $k$-block approximation of $\boldsymbol{x}$ as

$$
\boldsymbol{x}_{[k]}=\arg \min _{\|\boldsymbol{u}\|_{2,0} \leq k}\|\boldsymbol{x}-\boldsymbol{u}\|_{2,1}, \quad \boldsymbol{u} \in \mathbb{R}^{n}
$$

For convenience, in the remainder of this paper, we use $\delta_{k}$ and $\theta_{k_{1}, k_{2}}$, instead of $\delta_{k \mid d}$ and $\theta_{\left(k_{1}, k_{2}\right) \mid d}$, to represent the block $\Psi$-RIC and block $\Psi$-ROC, respectively, whenever confusion is not caused.

Next, we present our main results, which are included in the following theorem.

Theorem 1 Let $k_{1}$ and $k_{2}$ be two positive integers such that $0 \leq 8\left(k_{1}-k\right) \leq k_{2}$, and denote $t=2^{1 / q-1}\left(k_{1} / k_{2}\right)^{1 / q-1 / 2}+\sqrt{k_{2} / k_{1}}\left[(q / 2)^{q /(2-q)}-\right.$ $\left.(q / 2)^{2 /(2-q)}\right]-\left(1+2^{1 / q-1}\right) \sqrt{k_{2} / k_{1}}\left[\left(k_{1}-k\right) / k_{2}\right]^{1 / q}$ with $0<q \leq 1$. If the matrix $\Phi$ satisfies

$$
\delta_{k_{1}}+t \theta_{k_{1}, k_{2}}<1
$$

where $\delta_{k}$ and $\theta_{k, k}$ are defined in Definitions 1 and 2, then the solution $\boldsymbol{f}^{\sharp}$ to problem (1) obeys

$$
\left\|\boldsymbol{f}-\boldsymbol{f}^{\sharp}\right\|_{2} \leq C_{1} \epsilon+C_{2}\left\|\left(\Psi^{T} \boldsymbol{f}\right)_{[k]}\right\|_{2, q},
$$

where

$$
\begin{aligned}
C_{1}= & \frac{2 \sqrt{\left(1+\delta_{k_{1}}\right)\left(\left(2 k_{1}\right)^{1 / q-1}+1\right)}}{1-\delta_{k_{1}}-t \theta_{k_{1}, k_{2}}} \\
C_{2}= & \frac{2^{2 / q-1}\left(k_{2}\right)^{1 / 2-1 / q_{k_{1}, k_{2}}} \sqrt{\left(2 k_{1}\right)^{1 / q-1}+1}}{1-\delta_{k_{1}}-t \theta_{k_{1}, k_{2}}} \\
& +\frac{2^{2 / q-2}}{\sqrt{k_{1}\left(\left(2 k_{1}\right)^{1 / q-1}+1\right)}}
\end{aligned}
$$

In what follows, we present two remarks for the established results, and the proof of the theorem will be given in the appendix.

Remark 1 Theorem 1 presents a sufficient condition to robustly recover general signals via the $\ell_{2} / \ell_{q}$-analysis method with $0<q \leq 1$. The obtained sufficient condition associates block $\Psi$-RIC and block $\Psi$-ROC with different $q \in(0,1]$, and provides a series of selectable conditions for robust recovery of general signals that can be expressed as block-sparse vectors. Since condition (2) is related to $t$, which is a complex combination of $k_{1}, k_{2}, k$, and $q$, it is difficult to intuitively analyze the obtained condition. So by choosing some representative values, we induce a series of new conditions, as detailed in Table 1.

Remark 2 Inequality (3) indicates that our reconstructed error $\left\|\boldsymbol{f}-\boldsymbol{f}^{\sharp}\right\|_{2}$ can be bounded by the lowest $k$-block approximation error and the noise level $\epsilon$. As a special case where $\epsilon=0$ and the original signal $f$ can be expressed as a block $k$-sparse vector with a fixed $\Psi$, i.e., $\left\|\Psi^{T} \boldsymbol{f}\right\|_{2,0} \leq k$, if the matrix $\Phi$ satisfies (2), solving problem (1) will lead to exact recovery of the original signal $\boldsymbol{f}$.

\section{Numerical experiments and results}

In this section, we conduct some numerical experiments to evaluate the performance of our $\ell_{2} / \ell_{q}(0<q<1)$ analysis method. An IRLS algorithm is first proposed to solve the induced $\ell_{2} / \ell_{q}(0<q<1)$-analysis problem. We then compare our $\ell_{2} / \ell_{q}(0<q<1)$-analysis method with other analysis-style methods, including $\ell_{2} / \ell_{1}$-analysis [17] and $\ell_{q}(0<q \leq 1)$-analysis [26].

\subsection{An IRLS algorithm for $\ell_{2} / \ell_{q}$-analysis}

In order to solve the $\ell_{2} / \ell_{q}$-analysis problem (1) with $0<$ $q \leq 1$, we derive an efficient analysis-style IRLS algorithm. 
Table 1 Different sufficient conditions related to $k_{1}, k_{2}$, and $q$

\begin{tabular}{llllll}
\hline$k_{1}$ & $k_{2}$ & $q=0.1$ & $q=0.5$ & $q=0.7$ & $q=1$ \\
\hline$k$ & $3 k$ & $\delta_{k}+1.43 \theta_{k, 3 k}<1$ & $\delta_{k}+1.21 \theta_{k, 3 k}<1$ & $\delta_{k}+1.13 \theta_{k, 3 k}<1$ & $\delta_{k}+1.02 \theta_{k, 3 k}<1$ \\
$k$ & $4 k$ & $\delta_{k}+1.63 \theta_{k, 4 k}<1$ & $\delta_{k}+1.2 \theta_{k, 4 k}<1$ & $\delta_{k}+1.12 \theta_{k, 4 k}<1$ & $\delta_{k}+\theta_{k, 4 k}<1$ \\
$\frac{5}{4} k$ & $\frac{11}{4} k$ & $\delta_{k}+1.49 \theta_{\frac{5}{4} k, \frac{11}{4} k}<1$ & $\delta_{k}+1.28 \theta_{\frac{5}{4} k, \frac{11}{4} k}<1$ & $\delta_{k}+1.09 \theta_{\frac{5}{4} k, \frac{11}{4} k}<1$ & $\delta_{k}+0.78 \theta_{\frac{5}{4} k, \frac{11}{4} k}<1$ \\
$\frac{3}{2} k$ & $\frac{7}{2} k$ & $\delta_{k}+1.41 \theta_{\frac{3}{2} k, \frac{7}{2} k}<1$ & $\delta_{k}+1.19 \theta_{\frac{3}{2} k, \frac{7}{2} k}<1$ & $\delta_{k}+0.96 \theta_{\frac{3}{2} k, \frac{7}{2} k}<1$ & $\delta_{k}+0.61 \theta_{\frac{3}{2} k, \frac{7}{2} k}<1$ \\
$2 k$ & $5 k$ & $\delta_{k}+1.37 \theta_{2 k, 5 k}<1$ & $\delta_{k}+1.07 \theta_{2 k, 5 k}<1$ & $\delta_{k}+0.79 \theta_{2 k, 5 k}<1$ & $\delta_{k}+0.4 \theta_{2 k, 5 k}<1$ \\
\hline
\end{tabular}

The proposed algorithm can be seen as a natural extension of the traditional IRLS algorithm [21, 27] for sparse problems. We first rewrite the problem (1) as

$$
\min _{\boldsymbol{f} \in \mathbb{R}^{N}}\left\|\Psi^{T} \boldsymbol{f}\right\|_{2, q}^{\epsilon}+\frac{1}{2 \lambda}\|\boldsymbol{y}-\Phi \boldsymbol{f}\|_{2}^{2}
$$

where $\lambda$ is a regularization parameter and $\left\|\Psi^{T} \boldsymbol{f}\right\|_{2, q}^{\epsilon}=$ $\sum_{i=1}^{m}\left(\epsilon^{2}+\left\|\Psi[i]^{T} f\right\|_{2}^{2}\right)^{\frac{q}{2}}$.

Using the first-order optimality condition on (4), we have

$$
\sum_{i=1}^{m} \frac{q \Psi[i] \Psi[i]^{T}}{\left(\epsilon^{2}+\left\|\Psi[i]^{T} \tilde{\boldsymbol{f}}\right\|_{2}^{2}\right)^{1-q / 2}} \tilde{\boldsymbol{f}}+\frac{1}{\lambda}\left(\Phi^{T} \Phi \tilde{\boldsymbol{f}}-\Phi^{T} \boldsymbol{y}\right)=0,
$$

where $\tilde{\boldsymbol{f}}$ denotes a critical point of (4). Due to the nonlinearity in the above equation, there is no straightforward way to obtain an accurate solution of (5). However, utilizing some numerical techniques, one can well approximate an accurate solution of (5). Along the ideas in [21, 27], we present a similar iterative procedure as

$\left\{\sum_{i=1}^{m} \frac{q \lambda \Psi[i] \Psi[i]^{T}}{\left[\left(\epsilon^{(t)}\right)^{2}+\left\|\Psi[i]^{T} \boldsymbol{f}^{(t)}\right\|_{2}^{2}\right]^{1-q / 2}}+\Phi^{T} \Phi\right\} \boldsymbol{f}^{(t+1)}=\Phi^{T} \boldsymbol{y}$,

which is implemented by Algorithm 1 .

\subsection{Experimental settings}

Throughout the experiments, the measurement matrix $\Phi$ is generated by creating an $\widetilde{m} \times \widetilde{n}$ Gaussian matrix with $\widetilde{m}=64$ and $\widetilde{n}=256$, and the overcomplete and tight frame $\Psi$ is generated by taking the first $\tilde{n}$ rows from an $n \times n$ Hadamard matrix with $n=512$. The original signal $\boldsymbol{f}$ is synthesized as $\boldsymbol{f}=\Psi \boldsymbol{x}$ where $\boldsymbol{x}$ is a block $k$ sparse signal with block size $d=4$. We set the value of the noise vector $\boldsymbol{e}$ as obeying a Gaussian distribute with mean 0 and standard deviation 0.05 . We consider four different values of $q=0.1,0.5,0.7,1$ for both the $\ell_{2} / \ell_{q}$-analysis and $\ell_{q}$-analysis methods. The relative error between the reconstructed signal $\boldsymbol{f}^{\sharp}$ and the original signal $\boldsymbol{f}$ is calculated as $\left\|\boldsymbol{f}-\boldsymbol{f}^{\sharp}\right\|_{2} /\|\boldsymbol{f}\|_{2}$.

\subsection{Experimental results}

In order to find the value of $\lambda$ that minimizes the relative error, we conduct two sets of trials. Figure 1a depicts the relative error versus $\lambda$ for recovering the signals $f$, which can be expressed as block 5 -sparse signals in terms of $\Psi$. It is easy to see that choosing $\lambda$ less than $1 \times 10^{-2}$ is appropriate. Similar results can also be found in Fig. 1b. Without loss of generality, we take $\lambda=1 \times 10^{-3}$ as the best regularization parameter value.

Next, we compare our $\ell_{2} / \ell_{q}(0<q<1)$-analysis method with the $\ell_{2} / \ell_{1}$-analysis and $\ell_{q}(0<q \leq 1)$ analysis methods. The results are depicted in Fig. 2.

It is easy to see that the $\ell_{2} / \ell_{q}(0<q<1)$-analysis method is far superior to the $\ell_{2} / \ell_{1}$-analysis method. Take for example the $\ell_{2} / \ell_{0.5}$-analysis method when $k=8$. The relative error from the $\ell_{2} / \ell_{0.5}$-analysis method is 0.016 , which is about 16 times smaller than that of the $\ell_{2} / \ell_{1}$-analysis method (0.257). Additionally, in terms of the non-convex strategy, a proper value of $q$ contributes to better performance of both the $\ell_{2} / \ell_{q}(0<q \leq 1)$-analysis and $\ell_{q}(0<q \leq 1)$-analysis methods. However, with increasing block-sparsity $k$, the three methods above tend to consistency. What is more, when it comes to recovering the signals, which can be expressed as block-sparse coefficient vectors based on $\Psi$, our method performs better than the other two methods. An instance is also presented in Fig. 3, which displays the recovery of the signal $\boldsymbol{f}$ synthesized by a block 7 -sparse vector based on the $\Psi$ via $\ell_{2} / \ell_{q}(0<q \leq 1)$-analysis and $\ell_{q}(0<q \leq 1)$-analysis methods, respectively.

\section{Conclusion and discussion}

This paper mainly investigates an $\ell_{2} / \ell_{q}(0<q \leq 1)$ analysis method to recover a general signal that can be expressed as a block-sparse vector in terms of an overcomplete and tight frame. To the best of our knowledge, this is the first theoretical characterization of the proposed non-convex $\ell_{2} / \ell_{q}$-analysis method with $0<q<1$. Specifically, the obtained results contribute to CS in the following three aspects: 


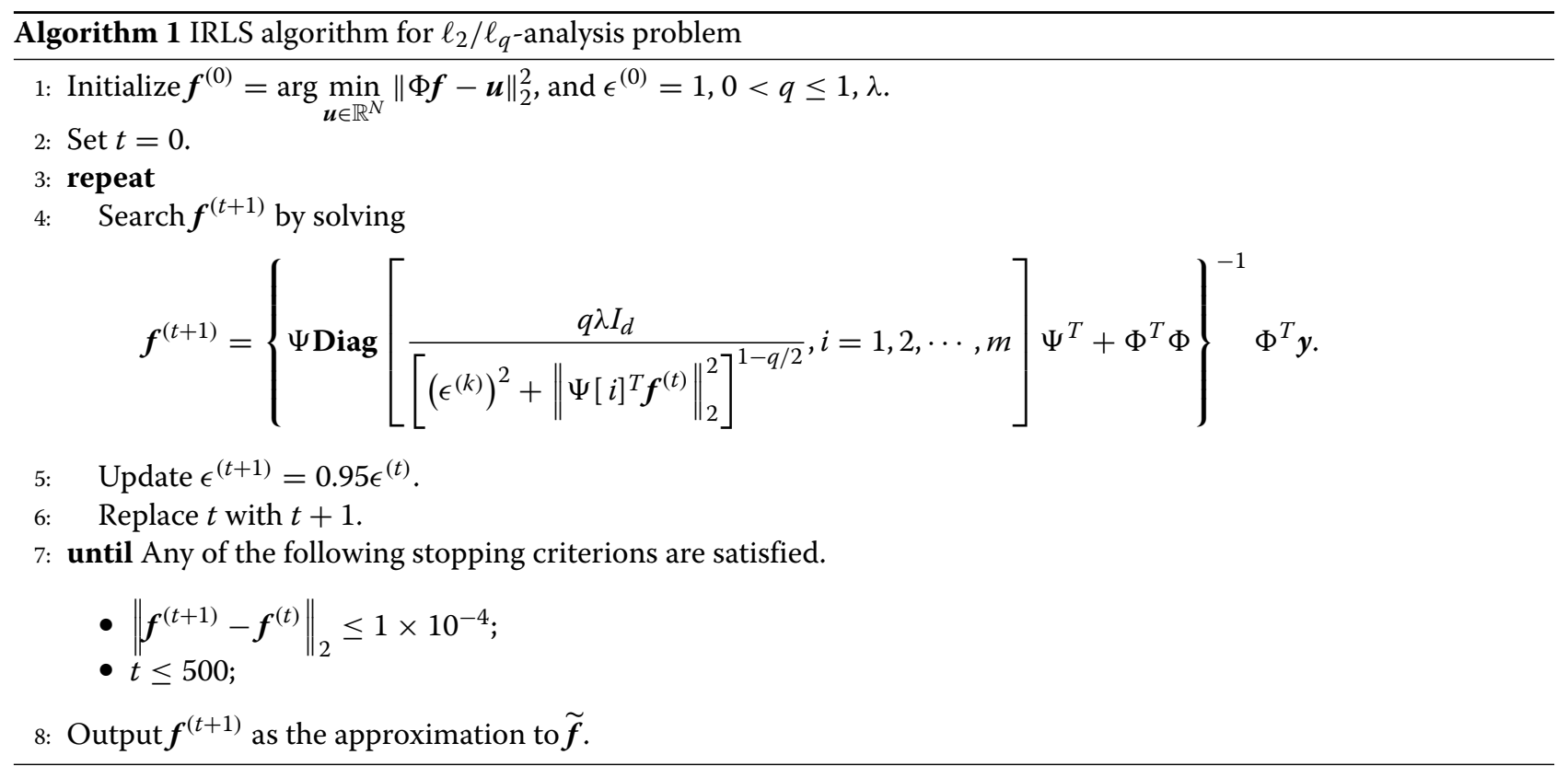

- We proposed an $\ell_{2} / \ell_{q}$-analysis method to recover a general signal that can be expressed as a block-sparse vector in a certain frame, generalizing both the traditional methods in CS to recover sparse signals and the recent novel analysis methods to recover general signals.

- Basing our theoretical approach on a proposed $\ell_{2} / \ell_{q}$-analysis method, we established a sufficient condition for robust recovery of general signals that can be expressed as a block-sparse signals, which associates block $\Psi$-RIC and block $\Psi$-ROC with different values of $q \in(0,1]$, providing a series of selectable conditions related to $q$.

- We derive an analysis-style IRLS algorithm to solve the proposed method and compare our method with that of other representative methods, obtaining some convincing results.

There are still some issues left for future work. For example, one could consider establishing sharp recovery conditions of our $\ell_{2} / \ell_{q}(0<q<1)$-analysis method, and one could also consider replacing our $\ell_{2} / \ell_{q}(0<q<$ $1)$-analysis method with other more general non-convex methods.

\section{Appendix}

The proof of Theorem 1 is proved as follows.

Let $\boldsymbol{f}^{\sharp}=\boldsymbol{f}+\boldsymbol{h}$ be a solution of (1), where $\boldsymbol{f}$ is the original signal. Write $\Psi^{T} \boldsymbol{h}=(\boldsymbol{c}[1], \boldsymbol{c}[2], \cdots, \boldsymbol{c}[m])^{T}$ and rearrange the block indices such that $\|\boldsymbol{c}[1]\|_{2} \geq\|\boldsymbol{c}[2]\|_{2} \geq$ $\cdots \geq\|c[m]\|_{2}$. Let $\widetilde{\Omega}=\{1,2, \cdots, k\}$ and $\Omega$ the block index set over the $k$ blocks with largest $\ell_{2}$ norm of $\Psi^{T} \boldsymbol{f}$. We denote by $\Omega^{\mathrm{C}}$ the complement set of $\Omega$ in $\{1,2, \cdots, d\}$. For convenience, we use $\Psi_{\widetilde{\Omega}}^{T}$ to denote $\left(\Psi_{\widetilde{\Omega}}\right)^{T}$ where $\Psi_{S}$ is the matrix $\Psi$ restricted to the column-blocks indexed by $\widetilde{\Omega}$, and then partition $\{1,2, \cdots, d\}$ into the following sets

$$
\begin{aligned}
& \Omega_{0}=\left\{1,2, \cdots, k_{1}\right\} \\
& \Omega_{1}=\left\{k_{1}+1, k_{1}+2, \cdots, k_{1}+k_{2}\right\}, \\
& \Omega_{2}=\left\{k_{1}+k_{2}+1, k_{1}+k_{2}+2, \cdots, k_{1}+2 k_{2}\right\}
\end{aligned}
$$

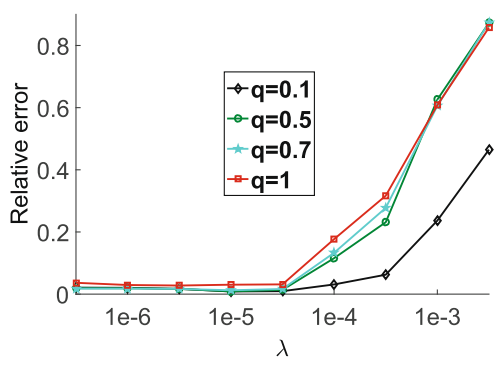

(a)

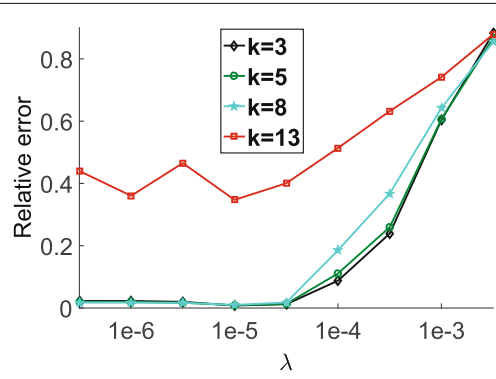

(b)

Fig. 1 Selection of regularization parameter $\lambda$ over the range from $1 \times 10^{-6}$ to 1 . $\mathbf{a} k=5 . \mathbf{b} q=0: 5$ 


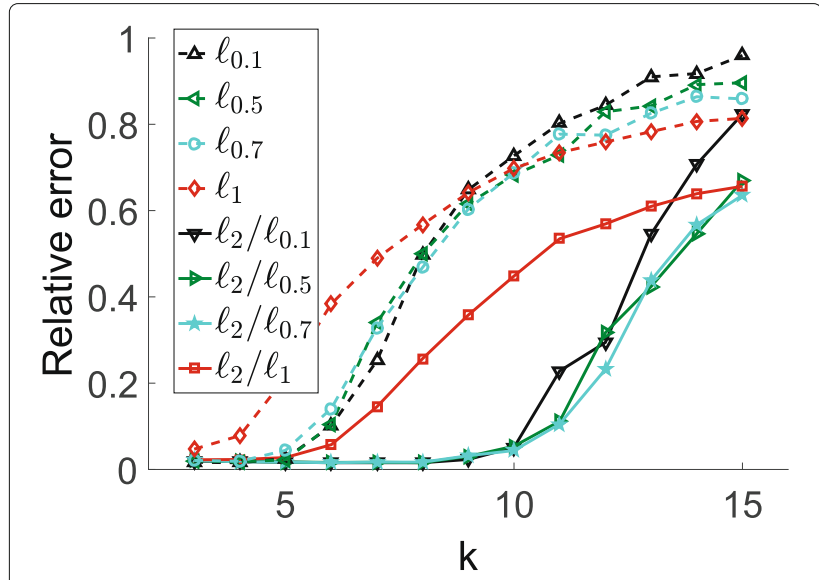

Fig. 2 Comparison of recovery performance among non-convex $\ell_{2} / \ell_{q}(0<q<1)$-analysis, $\ell_{2} / \ell_{1}$-analysis and $\ell_{q}(0<q \leq 1)$-analysis methods

Since $\boldsymbol{f}^{\sharp}$ is a minimizer of (1), we have

$$
\begin{aligned}
& \left\|\Psi_{\Omega}^{T} \boldsymbol{f}\right\|_{2, q}^{q}+\left\|\Psi_{\Omega^{c}}^{T} \boldsymbol{f}\right\|_{2, q}^{q} \\
& =\left\|\Psi^{T} \boldsymbol{f}\right\|_{2, q}^{q} \geq\left\|\Psi_{\Omega}^{T}(\boldsymbol{f}+\boldsymbol{h})\right\|_{2, q}^{q}+\left\|\Psi_{\Omega^{\mathrm{c}}}^{T}(\boldsymbol{f}+\boldsymbol{h})\right\|_{2, q}^{q} \\
& \geq\left\|\Psi_{\Omega}^{T} \boldsymbol{f}\right\|_{2, q}^{q}-\left\|\Psi_{\Omega}^{T} \boldsymbol{h}\right\|_{2, q}^{q}+\left\|\Psi_{\Omega^{\mathrm{c}}}^{T} \boldsymbol{h}\right\|_{2, q}^{q}-\left\|\Psi_{\Omega^{\mathrm{c}}}^{T} \boldsymbol{f}\right\|_{2, q}^{q} .
\end{aligned}
$$

This implies

$$
\left\|\Psi_{\Omega^{\mathrm{c}}}^{T} \boldsymbol{h}\right\|_{2, q}^{q} \leq 2\left\|\Psi_{\Omega^{\mathrm{c}}}^{T} \boldsymbol{f}\right\|_{2, q}^{q}+\left\|\Psi_{\Omega}^{T} \boldsymbol{h}\right\|_{2, q}^{q} .
$$

Note that $\left\|\Psi_{\widetilde{\Omega}}^{T} \boldsymbol{h}\right\|_{2, q} \geq\left\|\Psi_{\Omega}^{T} \boldsymbol{h}\right\|_{2, q}$ and $\left\|\Psi_{\widetilde{\Omega}^{\mathrm{c}}}^{T} \boldsymbol{h}\right\|_{2, q} \leq$ $\left\|\Psi_{\Omega^{\mathrm{c}}}^{T} \boldsymbol{h}\right\|_{2, q}$, and thus it follows from (6) that

$$
\left\|\Psi_{\widetilde{\Omega}^{\mathrm{c}}}^{T} \boldsymbol{h}\right\|_{2, q}^{q} \leq\left\|\Psi_{\widetilde{\Omega}}^{T} \boldsymbol{h}\right\|_{2, q}^{q}+2\left\|\Psi_{\Omega^{\mathrm{c}}}^{T} \boldsymbol{f}\right\|_{2, q}^{q} .
$$

Further, we have

$$
\left\|\Psi_{\widetilde{\Omega}^{\mathrm{c}}}^{T} \boldsymbol{h}\right\|_{2, q} \leq 2^{1 / q-1}\left\|\Psi_{\widetilde{\Omega}}^{T} \boldsymbol{h}\right\|_{2, q}+2^{2 / q-1}\left\|\Psi_{\Omega^{\mathrm{c}}}^{T} \boldsymbol{f}\right\|_{2, q}
$$

Using the inequality involving $\ell_{2}$ and $\ell_{q}(0<q \leq 1)$ norms $^{1}$ (see [28], Lemma 3), it is easy to obtain that

$$
\begin{aligned}
\left\|\Psi_{\Omega_{j}}^{T} \boldsymbol{h}\right\|_{2} & \leq\left(k_{2}\right)^{1 / 2-1 / q}\left\|\Psi_{\Omega_{j}}^{T} \boldsymbol{h}\right\|_{2, q} \\
& +P_{q} \sqrt{k_{2}}\left\{\left\|\boldsymbol{c}\left[k_{1}+(j-1) k_{2}+1\right]\right\|_{2}-\left\|\boldsymbol{c}\left[k_{1}+j k_{2}\right]\right\|_{2}\right\}
\end{aligned}
$$

holds for any $j \geq 1$, where $P_{q}=(q / 2)^{q /(2-q)}-(q / 2)^{2 /(2-q)}$. Thus, summing these terms yields

${ }^{1}$ In fact, when $0<q<1$, the $\ell_{q}$ norm is a quasi norm. For consistency, we instead use the norm.

$$
\begin{aligned}
\sum_{j \geq 1}\left\|\Psi_{\Omega_{j}}^{T} \boldsymbol{h}\right\|_{2} \leq & \left(k_{2}\right)^{1 / 2-1 / q} \sum_{j \geq 1}\left\|\Psi_{\Omega_{j}}^{T} \boldsymbol{h}\right\|_{2, q} \\
& +P_{q} \sqrt{k_{2}}\left\|\boldsymbol{c}\left[k_{1}+1\right]\right\|_{2} \\
\leq & \left(k_{2}\right)^{1 / 2-1 / q}\left\{\left\|\Psi_{\widetilde{\Omega}}^{T} \boldsymbol{h}\right\|_{2, q}-\left(k_{1}-k\right)^{1 / q}\left\|\boldsymbol{c}\left[k_{1}+1\right]\right\|_{2}\right\} \\
& +P_{q} \sqrt{k_{2}}\left\|\boldsymbol{c}\left[k_{1}+1\right]\right\|_{2} .
\end{aligned}
$$

This, along with (7), thus gives

$$
\begin{aligned}
\sum_{j \geq 1}\left\|\Psi_{\Omega_{j}}^{T} \boldsymbol{h}\right\|_{2} \leq & \left(k_{2}\right)^{1 / 2-1 / q}\left\|\Psi_{\Omega^{c}}^{T} \boldsymbol{h}\right\|_{2, q} \\
& +\left[P_{q} \sqrt{k_{2}}-\left(k_{2}\right)^{1 / 2-1 / q}\left(k_{1}-k\right)^{1 / q}\right]\left\|\boldsymbol{c}\left[k_{1}+1\right]\right\|_{2} \\
\leq & 2^{1 / q-1}\left(k_{2}\right)^{1 / 2-1 / q}\left\|\Psi_{\widetilde{\Omega}}^{T} \boldsymbol{h}\right\|_{2, q} \\
& +2^{2 / q-1}\left(k_{2}\right)^{1 / 2-1 / q}\left\|\Psi_{\Omega^{c}}^{T} \boldsymbol{f}\right\|_{2, q} \\
& +\left[P_{q} \sqrt{k_{2}}-\left(k_{2}\right)^{1 / 2-1 / q}\left(k_{1}-k\right)^{1 / q}\right]\left\|\boldsymbol{c}\left[k_{1}+1\right]\right\|_{2} \\
\leq & 2^{1 / q-1}\left(k_{2}\right)^{1 / 2-1 / q}\left\{\left\|\Psi_{\Omega_{0}}^{T} \boldsymbol{h}\right\|_{2, q}-\left(k_{1}-k\right)^{1 / q}\left\|\boldsymbol{c}\left[k_{1}+1\right]\right\|_{2}\right\} \\
& +2^{2 / q-1}\left(k_{2}\right)^{1 / 2-1 / q}\left\|\Psi_{\Omega^{c}}^{T} \boldsymbol{f}\right\|_{2, q} \\
& +\left[P_{q} \sqrt{k_{2}}-\left(k_{2}\right)^{1 / 2-1 / q}\left(k_{1}-k\right)^{1 / q}\right]\left\|\boldsymbol{c}\left[k_{1}+1\right]\right\|_{2} \\
= & 2^{1 / q-1}\left(k_{2}\right)^{1 / 2-1 / q}\left\|\Psi_{\Omega_{0}}^{T} \boldsymbol{h}\right\|_{2, q} \\
& +2^{2 / q-1}\left(k_{2}\right)^{1 / 2-1 / q}\left\|\Psi_{\Omega^{c}}^{T} \boldsymbol{f}\right\|_{2, q} \\
& +\left[P_{q} \sqrt{k_{2}}-\left(k_{2}\right)^{1 / 2-1 / q}\left(k_{1}-k\right)^{1 / q}\right. \\
& \left.-2^{1 / q-1}\left(k_{2}\right)^{1 / 2-1 / q}\left(k_{1}-k\right)^{1 / q}\right]\left\|\boldsymbol{c}\left[k_{1}+1\right]\right\|_{2} .
\end{aligned}
$$

Since $\left\|\Psi_{\Omega_{0}}^{T} \boldsymbol{h}\right\|_{2, q} \leq\left(k_{1}\right)^{1 / q-1 / 2}\left\|\Psi_{\Omega_{0}}^{T} \boldsymbol{h}\right\|_{2}$ and $\boldsymbol{c}\left[k_{1}+\right.$ 1] $\left\|_{2}^{2} \leq\right\| \Psi_{\Omega_{0}}^{T} \boldsymbol{h} \|_{2}^{2} / k_{1}$, we can get

$\sum_{j \geq 1}\left\|\Psi_{\Omega_{j}}^{T} \boldsymbol{h}\right\|_{2} \leq t\left\|\Psi_{\Omega_{0}}^{T} \boldsymbol{h}\right\|_{2}+2^{2 / q-1}\left(k_{2}\right)^{1 / 2-1 / q}\left\|\Psi_{\Omega^{\mathrm{c}}}^{T} \boldsymbol{f}\right\|_{2, q}$,

where $t=2^{1 / q-1}\left(k_{1} / k_{2}\right)^{1 / q-1 / 2}+P_{q} \sqrt{k_{2} / k_{1}}-(1+$ $\left.2^{1 / q-1}\right) \sqrt{k_{2} / k_{1}}\left[\left(k_{1}-k\right) / k_{2}\right]^{1 / q}$.

In fact, to make (8) work, one way is to set

$P_{q} \sqrt{k_{2} / k_{1}}-\left(1+2^{1 / q-1}\right) \sqrt{k_{2} / k_{1}}\left[\left(k_{1}-k\right) / k_{2}\right]^{1 / q} \geq 0$,

$$
\text { for all } 0<q \leq 1 \text {, }
$$

which is equivalent to

$$
0 \leq \frac{k_{1}-k}{k_{2}} \leq\left[\frac{(q / 2)^{q /(2-q)}-(q / 2)^{2 /(2-q)}}{2^{1 / q-1}+1}\right]^{q} .
$$

To this end, we have to estimate the minimal value of $f(q)=\left[\frac{(q / 2)^{q /(2-q)}-(q / 2)^{2 /(2-q)}}{2^{1 / q-1}+1}\right]^{q}$, where $0<q \leq 1$.

By means of mathematical skills, we can deduce that $f(q)$ arrives at its minimum value 0.125 when $q=1$. An auxiliary result is depicted in Fig. 4. 

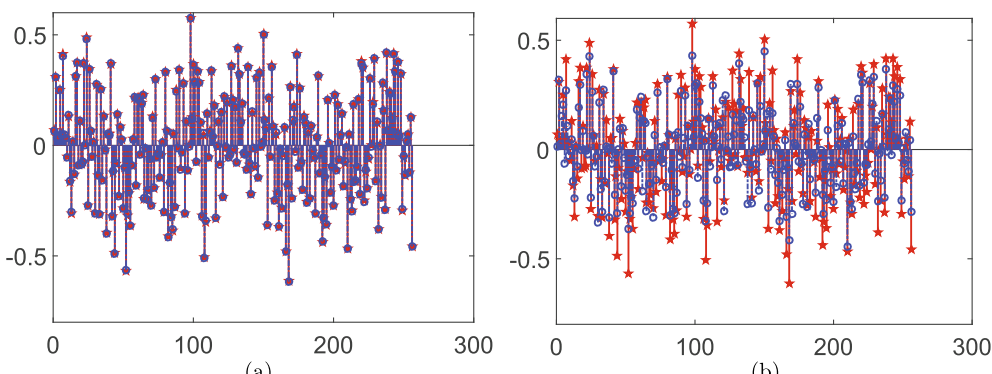

(b)

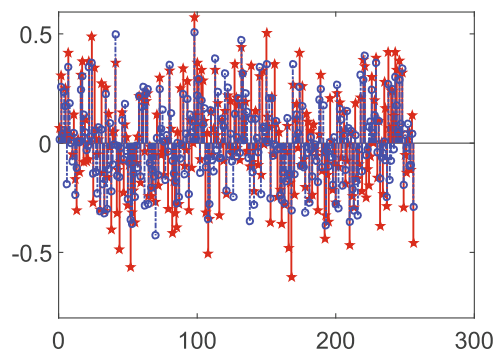

(c)

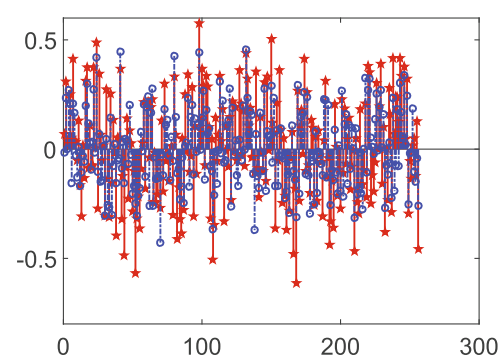

(d)

Fig. 3 An instance for recovering the signal, which can be expressed as a block 5-sparse vector in terms of $\Psi$ for different recovery methods. The solid line with pentagram markers represent the original signal, and the dotted line represents the recovered signal. a $\ell_{2} / \ell_{0.5}$-analysis method. b $\ell_{2} / \ell_{1}$-analysis method. $\mathbf{c} \ell_{0.5}$-analysis method. $\mathbf{d} \ell_{1}$-analysis method

A direct result is that if the condition $0 \leq \frac{k_{1}-k}{k_{2}} \leq 0.125$ holds, one can easily get (8) for all $0<q \leq 1$.

Similar to the consequence of $\Psi$-RIP, we have

$$
\begin{aligned}
\left\langle\Phi \boldsymbol{h}, \Phi \Psi \Psi_{\Omega_{0}}^{T} \boldsymbol{h}\right\rangle= & \left\|\Phi \Psi \Psi_{\Omega_{0}}^{T} \boldsymbol{h}\right\|_{2}^{2} \\
& +\sum_{j \geq 1}\left\langle\Phi \Psi \Psi_{\Omega_{j}}^{T} \boldsymbol{h}, \Phi \Psi \Psi_{\Omega_{0}}^{T} \boldsymbol{h}\right\rangle \\
\geq & \left(1-\delta_{k_{1}}\right)\left\|\Psi \Psi_{\Omega_{0}}^{T} \boldsymbol{h}\right\|_{2}^{2} \\
& +\sum_{j \geq 1}\left\langle\Psi \Psi_{\Omega_{j}}^{T} \boldsymbol{h}, \Psi \Psi_{\Omega_{0}}^{T} \boldsymbol{h}\right\rangle \\
& -\theta_{k_{1}, k_{2}}\left\|\Psi_{\Omega_{0}}^{T} \boldsymbol{h}\right\|_{2} \sum_{j \geq 1}\left\|\Psi_{\Omega_{j}}^{T} \boldsymbol{h}\right\|_{2} .
\end{aligned}
$$

By applying the equality

$$
\begin{aligned}
\sum_{j \geq 1}\left\langle\Psi \Psi_{\Omega_{j}}^{T} \boldsymbol{h}, \Psi \Psi_{\Omega_{0}}^{T} \boldsymbol{h}\right\rangle & =\left\langle\boldsymbol{h}-\Psi \Psi_{\Omega_{0}}^{T} \boldsymbol{h}, \Psi \Psi_{\Omega_{0}}^{T} \boldsymbol{h}\right\rangle \\
& =\left\|\Psi_{\Omega_{0}}^{T} \boldsymbol{h}\right\|_{2}^{2}-\left\|\Psi \Psi_{\Omega_{0}}^{T} \boldsymbol{h}\right\|_{2}^{2},
\end{aligned}
$$

to the above inequality, we get
$\left\langle\Phi \boldsymbol{h}, \Phi \Psi \Psi_{\Omega_{0}}^{T} \boldsymbol{h}\right\rangle$

$$
\begin{aligned}
\geq & \left\|\Psi_{\Omega_{0}}^{T} \boldsymbol{h}\right\|_{2}^{2}-\delta_{k_{1}}\left\|\Psi \Psi_{\Omega_{0}}^{T} \boldsymbol{h}\right\|_{2}^{2} \\
& -\theta_{k_{1}, k_{2}}\left\|\Psi_{\Omega_{0}}^{T} \boldsymbol{h}\right\|_{2} \sum_{j \geq 1}\left\|\Psi_{\Omega_{j}}^{T} \boldsymbol{h}\right\|_{2} \\
\geq & \left(1-\delta_{k_{1}}-t \theta_{k_{1}, k_{2}}\right)\left\|\Psi_{\Omega_{0}}^{T} \boldsymbol{h}\right\|_{2}^{2} \\
& -2^{2 / q-1}\left(k_{2}\right)^{1 / 2-1 / q} \theta_{k_{1}, k_{2}}\left\|\Psi_{\Omega_{0}}^{T} \boldsymbol{h}\right\|_{2}\left\|\Psi_{\Omega^{\mathrm{c}}}^{T} \boldsymbol{f}\right\|_{2, q} .
\end{aligned}
$$

By the feasibility of $\boldsymbol{f}^{\sharp}$, we have

$\|\Phi \boldsymbol{h}\|_{2}=\left\|\Phi\left(\boldsymbol{f}^{\sharp}-\boldsymbol{f}\right)\right\|_{2} \leq\left\|\Phi \boldsymbol{f}^{\sharp}-\boldsymbol{y}\right\|_{2}+\|\Phi \boldsymbol{f}-\boldsymbol{y}\|_{2} \leq 2 \epsilon$.

Thus,

$$
\begin{aligned}
\left\langle\Phi \boldsymbol{h}, \Phi \Psi \Psi_{\Omega_{0}}^{T} \boldsymbol{h}\right\rangle & \leq\|\Phi \boldsymbol{h}\|_{2}\left\|\Phi \Psi \Psi_{\Omega_{0}}^{T} \boldsymbol{h}\right\|_{2} \\
& \leq 2 \epsilon \sqrt{1+\delta_{k_{1}}}\left\|\Psi_{\Omega_{0}}^{T} \boldsymbol{h}\right\|_{2}
\end{aligned}
$$

It then follows from (10) and (11) that

$$
\begin{aligned}
\| & \Psi_{\Omega_{0}}^{T} \boldsymbol{h} \|_{2} \leq \frac{1}{1-\delta_{k_{1}}-t \theta_{k_{1}, k_{2}}} \\
& {\left[2 \epsilon \sqrt{1+\delta_{k_{1}}}+2^{2 / q-1}\left(k_{2}\right)^{1 / 2-1 / q} \theta_{k_{1}, k_{2}}\left\|\Psi_{\Omega^{\mathrm{c}}}^{T} \boldsymbol{f}\right\|_{2, q}\right] . }
\end{aligned}
$$




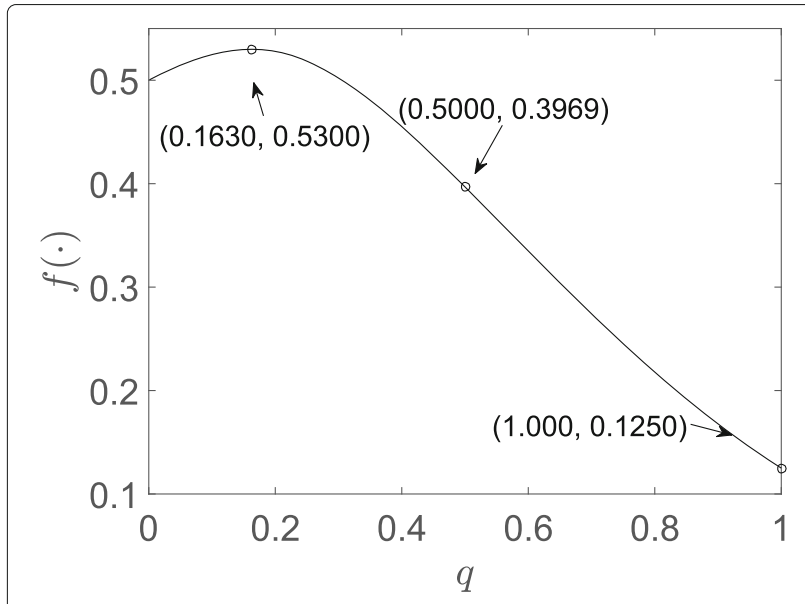

Fig. 4 Plot of $f(\cdot)$ (given in (9)) with respect to $q$ from 0.001 to 1 with step size 0.001 . One can easily find that the function $f(q)$ obtains its maximum (around 0.5300 ) at about $q=0.16$ and its minimum value (around 0.125 ) at $q=1$. Besides, when $q=0.5$ the value of $f(q)$ is around about 0.3969 . In other words, $f(q) \geq f(1)$ for any $q \in(0,1]$

\section{By (7), it is easy to see that}

$$
\begin{aligned}
\left\|\Psi_{\Omega_{0}} \mathrm{~h}\right\|_{2}^{2} \leq & \left\|\Psi_{\Omega_{0}} \mathrm{~h}\right\|_{2, q} \frac{\left\|\Psi_{\Omega_{0}}^{T} \boldsymbol{h}\right\|_{2,1}}{k_{1}} \\
& \leq\left\|\Psi_{\Omega^{c}} \boldsymbol{h}\right\|_{2, q} \frac{\left\|\Psi_{\Omega_{0}}^{T} \boldsymbol{h}\right\|_{2,1}}{k_{1}} \\
& \leq\left(2^{1 / q-1}\left\|\Psi_{\Omega_{0}}^{T} \boldsymbol{h}\right\|_{2, q}+2^{2 / q-1}\left\|\Psi_{\Omega^{c}}^{T} \boldsymbol{f}\right\|_{2, q}\right) \frac{\left\|\Psi_{\Omega_{0}}^{T} \boldsymbol{h}\right\|_{2,1}}{k_{1}} \\
& \leq\left(2 k_{1}\right)^{1 / q-1}\left\|\Psi_{\Omega_{0}}^{T} \boldsymbol{h}\right\|_{2}^{2}+\frac{2^{2 / q-1}}{\sqrt{k_{1}}}\left\|\Psi_{\Omega_{0}}^{T} \boldsymbol{h}\right\|_{2}\left\|\Psi_{\Omega^{c}}^{T} \boldsymbol{f}\right\|_{2, q} .
\end{aligned}
$$

Consequently, we have

$$
\begin{aligned}
\|\boldsymbol{h}\|_{2}^{2} & =\left\|\Psi^{T} \boldsymbol{h}\right\|_{2}^{2}=\left\|\Psi_{\Omega_{0}}^{T} \boldsymbol{h}\right\|_{2}^{2}+\left\|\Psi_{\widetilde{\Omega}^{c}}^{T} \boldsymbol{h}\right\|_{2}^{2} \\
& \leq\left[\left(2 k_{1}\right)^{1 / q-1}+1\right]\left\|\Psi_{\Omega_{0}}^{T} \boldsymbol{h}\right\|_{2}^{2}+\frac{2^{2 / q-1}}{\sqrt{k_{1}}}\left\|\Psi_{\Omega_{0}}^{T} \boldsymbol{h}\right\|_{2}\left\|\Psi_{\Omega^{c}}^{T} \boldsymbol{f}\right\|_{2, q} \\
& \leq\left\{\sqrt{\left(2 k_{1}\right)^{1 / q-1}+1}\left\|\Psi_{\Omega_{0}}^{T} \boldsymbol{h}\right\|_{2}+\frac{2^{2 / q-2}}{\sqrt{k_{1}\left[\left(2 k_{1}\right)^{1 / q-1}+1\right]}}\left\|\Psi_{\Omega^{c}}^{T} \boldsymbol{f}\right\|_{2, q}\right\}^{2} .
\end{aligned}
$$

Plugging (12) into the previously mentioned inequality and by a direct calculation, we get

$$
\begin{aligned}
\|\boldsymbol{h}\|_{2} \leq & \left\{\frac{2^{2 / q-1}\left(k_{2}\right)^{1 / 2-1 / q_{k_{1}, k_{2}}} \sqrt{\left(2 k_{1}\right)^{1 / q-1}+1}}{1-\delta_{k_{1}}-t \theta_{k_{1}, k_{2}}}\right. \\
& \left.+\frac{2^{2 / q-2}}{\sqrt{k_{1}\left[\left(2 k_{1}\right)^{1 / q-1}+1\right]}}\right\}\left\|D_{\Omega^{c}}^{T} \boldsymbol{f}\right\|_{2, q} \\
& +\frac{2 \sqrt{\left(1+\delta_{k_{1}}\right)\left[\left(2 k_{1}\right)^{1 / q-1}+1\right]}}{1-\delta_{k_{1}}-t \theta_{k_{1}, k_{2}}} \epsilon,
\end{aligned}
$$

which yields (3).

\section{Abbreviations}

$\Psi$-RIC: $\Psi$-Restricted isometry constant; $\Psi$-RIP: $\Psi$-Restricted isometry property; $\Psi$-ROC: $\Psi$-Restricted orthogonality constant; $\Psi$-ROP: $\Psi$-Restricted orthogonality property; CS: Compressed sensing; DNA: Deoxyribonucleic acid; IRLS: Iterative re-weighted least square

\section{Acknowledgments}

This paper is subsidized by the project of the key laboratory of Intelligent Information and Big Data Processing of NingXia Province, North Minzu University (no. NXKLIIBDP2019).

\section{Authors' contributions}

The authors read and approved the final manuscript.

\section{Availability of data and materials}

Please contact any of the authors for data and materials.

\section{Competing interests}

The authors declare that they have no competing interests.

\section{Author details}

${ }^{1}$ Faculty of Computer and Information Science, Southwest University, Chongqing 400715, China. ${ }^{2}$ Emergency Services, General Hospital of Ningxia Medical University, Yinchuan 750001, China. ${ }^{3}$ School of Mathematics and Information Science, North Minzu University, Yinchuan 750021, China. ${ }^{4}$ Faculty of Eduction, Southwest University, Chongqing 400715, China. ${ }^{5}$ Institute of Ethnic Preparatory Education, NingXia University, Yinchuan 750002, China.

Received: 29 July 2019 Accepted: 23 December 2019

Published online: 23 January 2020

\section{References}

1. D. Salomon, A concise introduction to data compression (2008)

2. D. Sculley, C. E. Brodley, in Proc. of the 2006 Data Compression Conference (DCC'2006). Compression and machine learning: A new perspective on feature space vectors (IEEE Computer Society, Snowbird, 2006), pp. 332-332. https://doi.org/10.1109/DCC.2006.13

3. L. Martino, V. Elvira, Compressed Monte Carlo for distributed Bayesian inference. viXra:1811.0505 (2018). https://www.rxiv.org/pdf/1811.0505v1. pdf

4. Y. Zheng, J. Ma, L. Wang, Consensus of hybrid multi-agent systems. IEEE Transactions on Neural Networks and Learning Systems. 29(4), 1359-1365 (2018)

5. J. Ma, M. Ye, Y. Zheng, Y. Zhu, Consensus analysis of hybrid multi-agent systems: a game-theoretic approach. Int. J. Robust Nonlinear Control. 29(6), 1840-1853 (2019)

6. D. L. Donoho, Compressed sensing. IEEE Trans. Inf. Theory. 52(4), 1289-1306 (2006)

7. E. J. Candès, J. Romberg, T. Tao, Robust uncertainty principles: Exact signal reconstruction from highly incomplete frequency information. IEEE Trans. Inf. Theory. 52(2), 489-509 (2006)

8. S. S. Chen, D. L. Donoho, M. A. Saunders, Atomic decomposition by basis pursuit. SIAM J. Sci. Comput. 20(1), 33-61 (1998)

9. A. M. Bruckstein, D. L. Donoho, M. Elad, From sparse solutions of systems of equations to sparse modeling of signals and images. SIAM Rev. 51(1), 34-81 (2009)

10. M. Elad, P. Milanfar, R. Rubinstein, Analysis versus synthesis in signal priors. Inverse Probl. 23(3), 947-968 (2007)

11. H. Rauhut, K. Schnass, P. Vandergheynst, Compressed sensing and redundant dictionaries. IEEE Trans. Inf. Theory. 54(5), 2210-2219 (2008)

12. E. J. Candès, Y. C. Eldar, D. Needel, Compressed sensing with coherent and redundant dictionaries. Appl. Comput. Harmon. Anal. 31 (1), 59-73 (2011)

13. I. Selesnick, M. Figueiredo, Signal restoration with overcomplete wavelet transforms: Comparison of analysis and synthesis priors. Proc. SPIE. 7446 (2009)

14. F. Parvaresh, H. Vikalo, S. Misra, B. Hassibi, Recovering sparse signals using sparse measurement matrices in compressed DNA microarrays. IEEE J. Sel. Top. Sig. Process. 2(3), 275-285 (2008) 
15. A. Majumdar, R. K. Ward, Compressed sensing of color images. Sig. Process. 90(12), 3122-3127 (2010)

16. R. Vidal, Y. Ma, A unified algebraic approach to 2-D and 3-D motion segmentation and estimation. J. Math. Imaging Vision. 25(3), 403-421 (2006)

17. Y. Wang, J. J. Wang, Z. B. Xu, A note on block-sparse signal recovery with coherent tight frames. Discret. Dyn. Nat. Soc. 2013(1), 1-8 (2013)

18. R. Chartrand, Exact reconstruction of sparse signals via nonconvex minimization. Sig. Process. Lett. 14(10), 707-710 (2007)

19. R. Chartrand, V. Staneva, Restricted isometry properties and nonconvex compressive sensing. Inverse Probl. 24(3), 20-35 (2008)

20. Z. B. Xu, H. L. Guo, Y. Wang, H. Zhang, Representative of $L_{1 / 2}$ regularization among $L_{q}(0<q \leq 1)$ regularizations: an experimental study based on phase diagram. Acta Autom. Sin. 38(7), 1225-1228 (2012)

21. Y. Wang, J. J. Wang, Z. B. Xu, On recovery of block-sparse signals via mixed $\ell_{2} / \ell_{q}(0<q \leq 1)$ norm minimization. EURASIP J. Adv. Sig. Process. 2013(1), 1-17 (2013)

22. Y. Wang, J. J. Wang, Z. B. Xu, Restricted p-isometry properties of nonconvex block-sparse compressed sensing. Sig. Process. 104, 188-196 (2014)

23. H. T. Yin, S. T. Li, L. Y. Fang, Block-sparse compressed sensing: non-convex model and iterative re-weighted algorithm. Inverse Probl. Sci. Eng. 21(1), $141-154$ (2013)

24. J. H. Lin, S. Li, Y. Shen, New bounds for restricted isometry constants with coherent tight frames. IEEE Trans. Sig. Process. 61(3), 611-621 (2013)

25. J. H. Lin, S. Li, Y. Shen, Compressed data separation with redundant dictionaries. IEEE Trans. Inf. Theory. 59(7), 4309-4315 (2013)

26. S. Li, J. H. Lin, Compressed sensing with coherent tight frame via $\ell_{q}$ minimization. Inverse Probl. Imaging. 8, 761-777 (2014)

27. M. J. Lai, Y. Y. Xu, W. T. Yin, Improved iteratively reweighted least squares for unconstrained smoothed $\ell_{q}$ minimization. SIAM J. Numer. Anal. 51(2), 927-957 (2013)

28. Y. Hsia, R. L. Sheu. arXiv: 1312.3379 (2014). http://arxiv.org/abs/1312.3379

\section{Publisher's Note}

Springer Nature remains neutral with regard to jurisdictional claims in published maps and institutional affiliations.

\section{Submit your manuscript to a SpringerOpen ${ }^{\circ}$ journal and benefit from:}

- Convenient online submission

- Rigorous peer review

- Open access: articles freely available online

- High visibility within the field

- Retaining the copyright to your article

Submit your next manuscript at $\gg$ springeropen.com 